\title{
Trop-2 is up-regulated in invasive prostate cancer and displaces FAK from focal contacts
}

\author{
Marco Trerotola ${ }^{1,2,6}$, Kirat K. Ganguly ${ }^{1,2}$, Ladan Fazli ${ }^{3}$, Carmine Fedele ${ }^{1,2}$, Huimin \\ Lu $^{1,2}$, Anindita Dutta ${ }^{1,2}$, Qin Liu ${ }^{1,4}$, Tiziana De Angelis ${ }^{1,2}$, Luke W. Riddell ${ }^{1,2}$, Natalia \\ A. Riobo ${ }^{5}$, Martin E. Gleave ${ }^{3}$, Amina Zoubeidi ${ }^{3}$, Richard G. Pestell ${ }^{2}$, Dario C. Altieri ${ }^{1,4}$ \\ and Lucia R. Languino ${ }^{1,2}$ \\ 1 Prostate Cancer Discovery and Development Program, Thomas Jefferson University, Philadelphia, PA, USA \\ 2 Department of Cancer Biology, Sidney Kimmel Cancer Center, Thomas Jefferson University, Philadelphia, PA, USA \\ ${ }^{3}$ The Vancouver Prostate Centre, University of British Columbia, Vancouver, British Columbia, Canada \\ ${ }^{4}$ Tumor Microenvironment and Metastasis Program, The Wistar Institute Cancer Center, Philadelphia, PA, USA \\ ${ }^{5}$ Department of Biochemistry, Thomas Jefferson University, Philadelphia, PA, USA \\ ${ }^{6}$ Current address: Ce.S.I. - University of Chieti-Pescara, Chieti Scalo, Italy \\ Correspondence to: Lucia R. Languino, email: lucia.languino@jefferson.edu
}

Keywords: PT2/PT3/pT4 prostate cancer, metastasis, gleason grade, TRAMP, exosome

Received: December 30, $2014 \quad$ Accepted: April 10, $2015 \quad$ Published: April 29, 2015

This is an open-access article distributed under the terms of the Creative Commons Attribution License, which permits unrestricted use, distribution, and reproduction in any medium, provided the original author and source are credited.

\section{ABSTRACT}

In this study, we show that the transmembrane glycoprotein Trop-2 is upregulated in human prostate cancer (PCa) with extracapsular extension (stages PT3/pT4) as compared to organ-confined (stage pT2) PCa. Consistent with this evidence, Trop-2 expression is found to be increased in metastatic prostate tumors of Transgenic Adenocarcinoma of Mouse Prostate mice and to strongly correlate with a5 $\beta 1$ integrin levels. Using PCa cells, we show that Trop-2 specifically associates with the a5 integrin subunit, as binding to a3 is not observed, and that Trop-2 displaces focal adhesion kinase from focal contacts. In support of the role of Trop-2 as a promoter of PCa metastatic phenotype, we observe high expression of this molecule in exosomes purified from Trop-2-positive PCa cells. These vesicles are then found to promote migration of Trop-2-negative PCa cells on fibronectin, an a5 31 integrin/ focal adhesion kinase substrate, thus suggesting that the biological function of Trop2 may be propagated to recipient cells. In summary, our findings show that Trop-2 promotes an a5 $\beta 1$ integrin-dependent pro-metastatic signaling pathway in PCa cells and that the altered expression of Trop- 2 may be utilized for early identification of capsule-invading PCa.

\section{INTRODUCTION}

The molecular mechanisms underlying the early phases of tumor invasion are not completely understood, although it is largely believed that acquisition of enhanced capacity to migrate through the extracellular matrix $(\mathrm{ECM})$ is a critical step for the onset of the metastatic cascade. When diagnosed at a non-invasive stage, prostate cancer $(\mathrm{PCa})$ is generally curable by surgical removal of the prostate gland. However, when PCa cells acquire the ability to break through the external capsule and invade the surrounding tissues, the chances to eradicate the disease by radical prostatectomy are reduced, resulting in lower overall survival rates for patients with metastatic disease. Widely accepted tumor staging criteria establish that stage pT2 identifies PCa still confined within the prostatic gland, whereas stages pT3/pT4 refer to PCa that has spread through the capsule and has eventually invaded adjacent structures [1,2]. Traditional disease monitoring approaches, including circulating prostate specific antigen (PSA) levels or Gleason scoring, do not discriminate between stage pT2 and stages pT3/pT4 [1, 2], thus hampering a central tenet for cancer patient stratification [3]. Therefore, developing molecular biomarkers that 


\begin{tabular}{|c|c|c|c|}
\hline \multirow[t]{2}{*}{ Score } & $\begin{array}{l}\text { pT2 } \\
(N=104)\end{array}$ & $\begin{array}{l}\text { pT3/pT4 } \\
(\mathrm{N}=44)\end{array}$ & $P$ \\
\hline & $n(\%$ of $N)$ & $n(\%$ of $N)$ & \\
\hline Trop-2 IHC Score & & & 0.0002 \\
\hline$<1.5$ & $73(70.2)$ & $8(18.2)$ & \\
\hline$\geq 1.5$ & $31(29.8)$ & $36(81.8)$ & \\
\hline Gleason Score & & & 0.0940 \\
\hline $6-7$ & $72(69.2)$ & 24 (54.6) & \\
\hline $8-10$ & $32(30.8)$ & $20(45.4)$ & \\
\hline \multicolumn{4}{|c|}{$\begin{array}{l}\text { Expression of Trop- } 2 \text { was evaluated by IHC using } 148 \text { PCa specimens as described in Methods. Samples were categorized } \\
\text { in groups based on Trop- } 2 \text { expression measured using IHC }(<1.5 \text { and } \geq 1.5) \text { or Gleason }(6-7 \text { and } 8-10) \text { scores. } P, P \text { value } \\
\text { was determined as described in the Methods section. }\end{array}$} \\
\hline
\end{tabular}

could identify specific stages of PCa progression remains an urgent, unmet medical need.

Previous studies have reported that the expression profile of many integrins, receptors for ECM substrates, becomes aberrant during cancer progression $[4,5]$. In particular, the $\alpha 5 \beta 1$ integrin heterodimer plays a pivotal role in development and progression of several types of carcinomas, including PCa [6, 7], and its expression correlates with reduced disease-free survival in several malignancies [7-9]. The $\alpha 5 \beta 1$ integrin is implicated in cell proliferation and growth [10]. A function-blocking antibody against $\alpha 5 \beta 1$ integrin significantly reduces tumor burden and metastasis in ovarian cancer models [7]. Additional studies demonstrate that the $\alpha 5 \beta 1$ integrin directly supports cell migration/invasion and metastasis $[11,12]$.

Metastatic dissemination is also promoted by exosomes, vesicles of endosomal origin, which are believed to generate a suitable microenvironment in the pre-metastatic niche $[13,14]$ by mediating horizontal transfer of genetic material [15] as well as of signaling molecules [14].

The epithelial transmembrane glycoprotein Trop2 functions as a key regulator of $\beta 1$ integrin activities by inducing cell detachment from ECM substrates and promoting motility of $\mathrm{PCa}$ cells $[16,17]$. Trop2 overexpression has been consistently linked to poor prognosis in many human cancers [18-21], suggesting a potential role of this molecule in metastatic dissemination. Specifically, we have previously shown that Trop-2 inhibits localization of $\beta 1$ integrins in focal adhesions (FAs) and induces hyperphosphorylation of focal adhesion kinase (FAK), deregulating cell-ECM interactions [17].

Altogether, the experimental findings presented here show that Trop-2 is a novel marker of capsule-invasive $\mathrm{PCa}$, is found in $\mathrm{PCa}$ cell exosomes and may function as mediator of PCa cell motility and metastasis.

\section{RESULTS}

\section{Trop-2 expression is increased in stages pT3/pT4 of human PCa}

Our previous findings demonstrate a role of Trop2 as an anti-adhesive and pro-migratory regulator in $\mathrm{PCa}$ $[16,17]$. Here, we hypothesized that up-regulation of Trop-2 promotes escape of $\mathrm{PCa}$ cells from the primary tumor microenvironment and accelerates the onset of the metastatic cascade. Hence, we performed an immunofluorescence (IF) analysis of human PCa tissues; as depicted in Figure 1A, an abundant distribution of Trop- 2 is found in membrane rims of the transformed cell population, whereas no reactivity is detected in the stromal compartment. We next analyzed Trop-2 expression levels in human PCa tissues by immunohistochemistry (IHC) using a Tissue Microarray (TMA) containing 104 cores from stage pT2 (organ-confined) and 44 cores from stages pT3/pT4 (PCa with extracapsular extension) cancer specimens collected from radical prostatectomies (Table 1). An example of Trop- 2 expression in stages $\mathrm{pT} 3$ and pT2 of PCa is shown in Figure 1B. The expression of Trop-2 in these specimens was evaluated as low (IHC score $<1.5$ ) or high (IHC score $\geq 1.5$ ) and is reported in Table 1. In these experiments, we observe strong expression of Trop- 2 in $29.8 \%$ of pT 2 stage and $81.8 \%$ in pT3/pT4 stage samples (Fisher's exact test $P=0.0002$ ). We analyzed in parallel the frequency of cases with high Gleason score (8-10) in stage pT2 and stages pT3/pT4 and found that the differences are not statistically significant (Fisher's exact test $P=0.0940$ ). Our results show that 
Trop-2 expression correlates with the stages pT3/pT4 in extracapsular invasive human PCa.

Trop-2 is up-regulated in prostate tumors of metastatic Transgenic Adenocarcinoma of Mouse Prostate (TRAMP) mice and forms a complex with the $\alpha 5 \beta 1$ integrin in PCa cells

Although mouse models of spontaneous $\mathrm{PCa}$ progression to metastasis are limited [22], the TRAMP model is known to develop aggressive and metastatic PCa [23]. We observe Trop-2 expression in metastatic prostate tumors of TRAMP mice using IF staining of prostate tumor tissue sections (Figure 2A). Macroscopic organ dissection of TRAMP mice $(n=69)$ was performed and primary tumors as well as metastases were analyzed. Figure 2B shows a representative normal genito-urinary (GU) (top left panel) and a primary tumor (bottom left panel), and also metastases in lung (top right panel) and liver (bottom right panel). Hematoxylin and Eosin (H\&E) analysis of non-metastatic (top left panel) and metastatic (bottom left panel) primary tumors is shown in Figure 2C. Analysis of lung (Figure 2C, top right panel) and liver (Figure 2C, bottom right panel) metastases are also shown.
We next analyzed the expression levels of Trop2 and of $\alpha 5, \beta 1, \beta 5$, and $\alpha \mathrm{v}$ integrin subunits in this experimental model, and compared metastatic $(n=4)$ with non-metastatic primary tumors $(n=4)$. As shown in Figure 3A, Trop-2 is highly expressed in metastatic tumor samples, but it is undetectable or expressed at low levels in non-metastatic tumors. Similarly, both $\alpha 5$ and $\beta 1$ integrin subunits are strongly up-regulated in metastatic prostate tumors as compared with non-metastatic tumors. These changes are found to be specific, as $\beta 5$, another integrin subunit, does not show appreciable variations in expression between metastatic and non-metastatic tumors. The $\alpha$ v integrin subunit, which does not associate with Trop-2 in PCa cells [16], was also preferentially expressed in metastatic tumors, suggesting the existence of additional regulatory mechanisms of this integrin subunit in $\mathrm{PCa}$.

Since Trop- 2 inhibits $\beta 1$ integrin-mediated PCa cell adhesion to fibronectin (FN) [17] and induces migratory phenotypes on this ECM ligand [16], we tested the ability of Trop-2 to interact with $\alpha 5 \beta 1$. Co-immunoprecipitation experiments performed using PC3 human PCa cells demonstrate that Trop-2 specifically associates with the $\alpha 5$ integrin subunit (Figure 3B). In contrast, another $\beta 1$ associated subunit, $\alpha 3$, does not interact with Trop-2 (Figure $3 \mathrm{C}$ ). These results provide a biochemical basis for
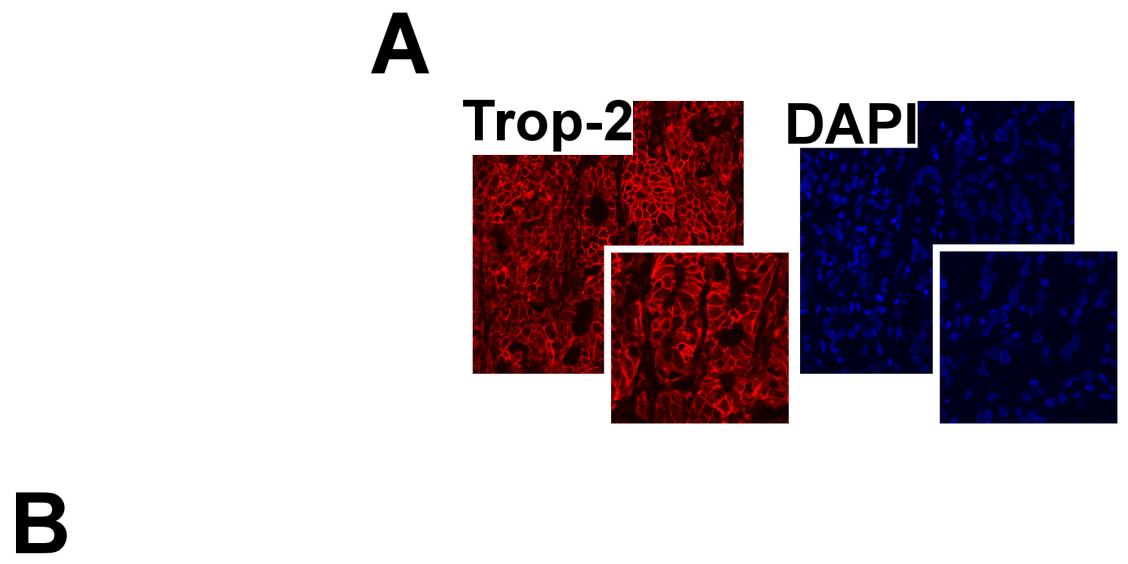

Trop-2

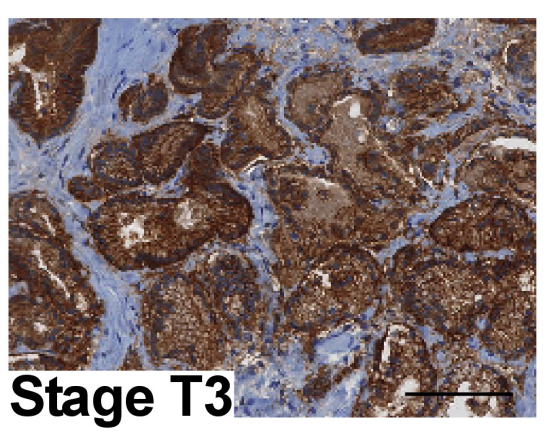

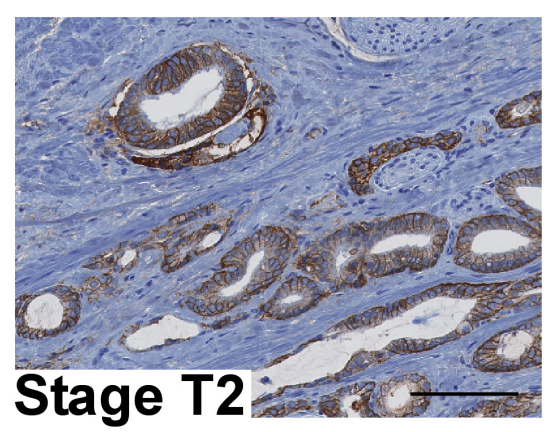

Neg. Ctr. IgG

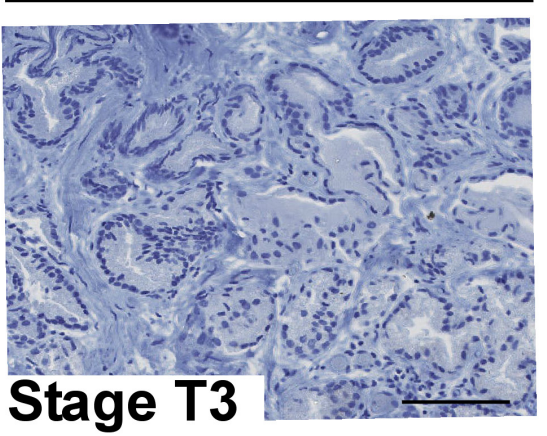

Figure 1: Trop-2 localization and expression in PCa. A. Localization of Trop-2 as investigated by IF staining and confocal microscopy in human PCa (pT3 stage, Gleason Score 9). B. Representative IHC staining for Trop-2 using specimens from patients at pT3 (left) and pT2 (middle) stages of PCa is shown. A non-immune IgG was used as negative control on a stage pT3 section (right). Bars, 100 $\mu \mathrm{m}$. 
the ability of Trop- 2 to specifically regulate $\alpha 5 \beta 1$ integrin functions.

\section{Trop-2 displaces focal adhesion kinase from focal contacts}

Recent findings from our group have shown that Trop- 2 inhibits accumulation of $\alpha 5 \beta 1$ integrin at FA sites [16], and promotes FAK activation [17]. To test this model, we stably silenced the expression of Trop- 2 in PCa cells and looked at the dynamics of FAK subcellular distribution. As shown in Figure 4 (right panel), the average number of FAK-containing FA sites is found to be
$178.60 \pm 0.53$ per cell in PC3/Trop-2 shRNA cells (FAKcontaining FAs, $n=5,358 / 30$ cells) as compared with $30.57 \pm 0.4$ per cell in PC3/control shRNA (Ctr.shRNA) cells (FAK-containing FAs, $n=917 / 30$ cells). Conversely, the average number of vinculin-containing FAs is 107.87 \pm 0.50 per cell in PC3/Trop- 2 shRNA cells (FAs, $n=$ $3,236 / 30$ cells) as compared with $110.53 \pm 0.43$ per cell in PC3/Ctr.shRNA cells (FA, $n=3,316 / 30$ cells), confirming the specificity of the observed response (Figure 4).

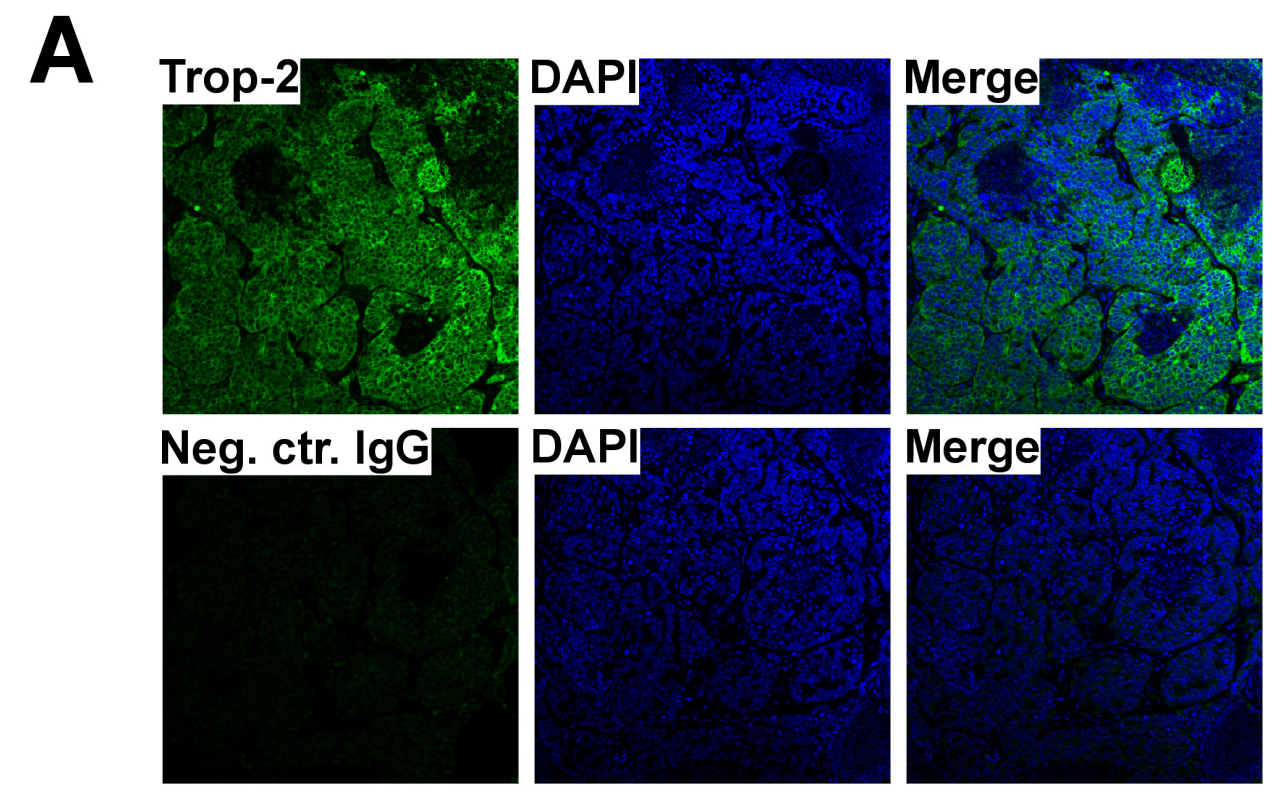

B

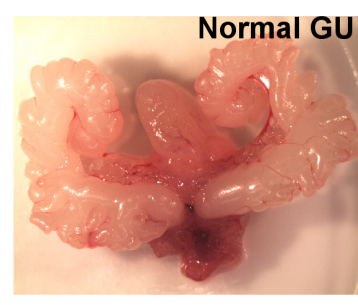

Prostate tumor

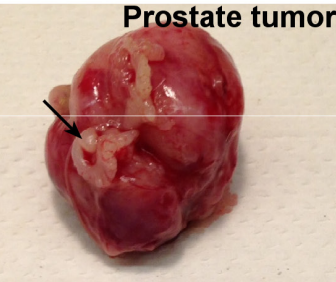

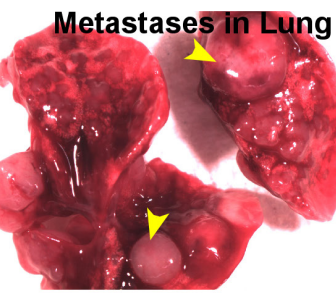

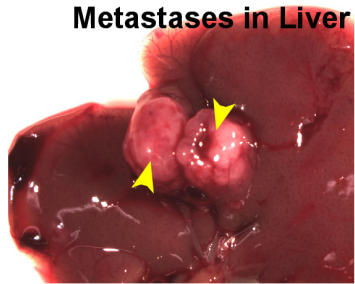

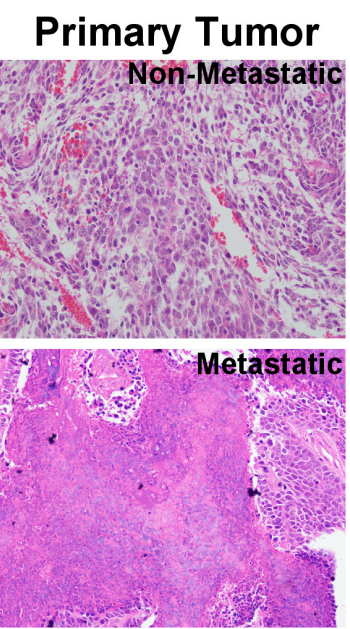

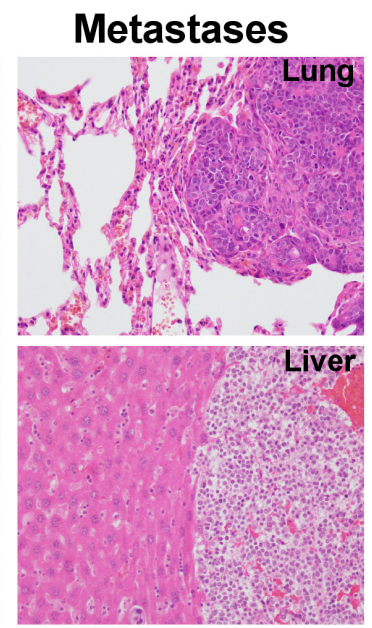

Figure 2: Analysis of Trop-2 expression in metastatic PCa from TRAMP mice. A. IF analysis of Trop-2 expression in metastatic prostate tumors from TRAMP mice (top). Cell nuclei were counterstained with DAPI. A non-immune goat IgG was used as a negative control Ab (bottom). B. Representative images of a dissected normal genito-urinary $(\mathrm{GU})$ system (top left), primary prostate tumor (bottom left), and lung (top right) and liver (bottom right) macroscopic metastases. Seminal vesicles (black arrow); metastases (yellow arrowheads). C. H\&E staining of non-metastatic (top left), metastatic primary prostate tumors (bottom left), and of metastases in lungs (top right) and liver (bottom right). 
A
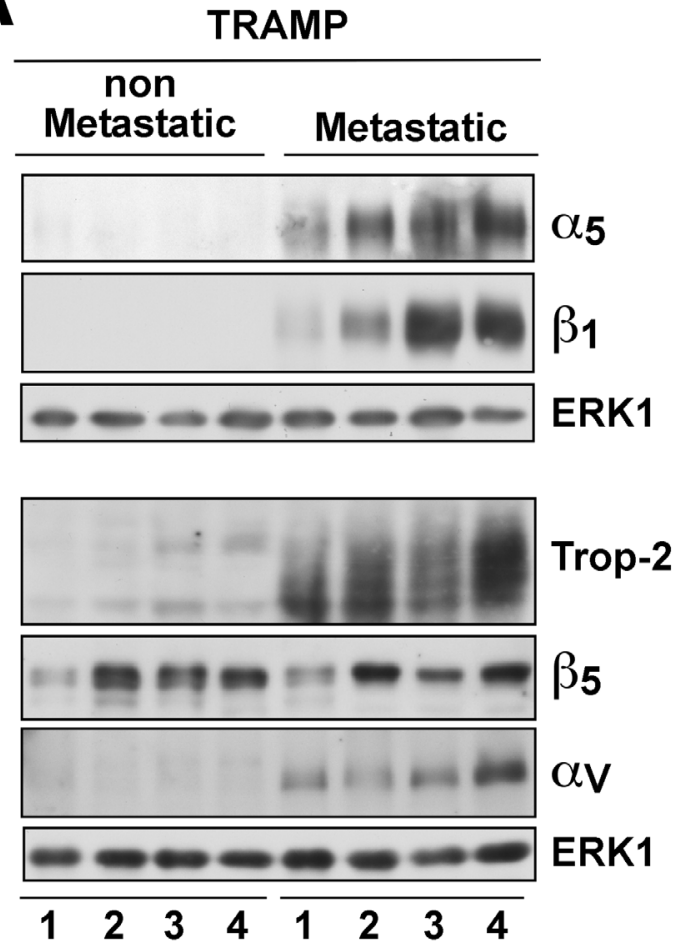

B
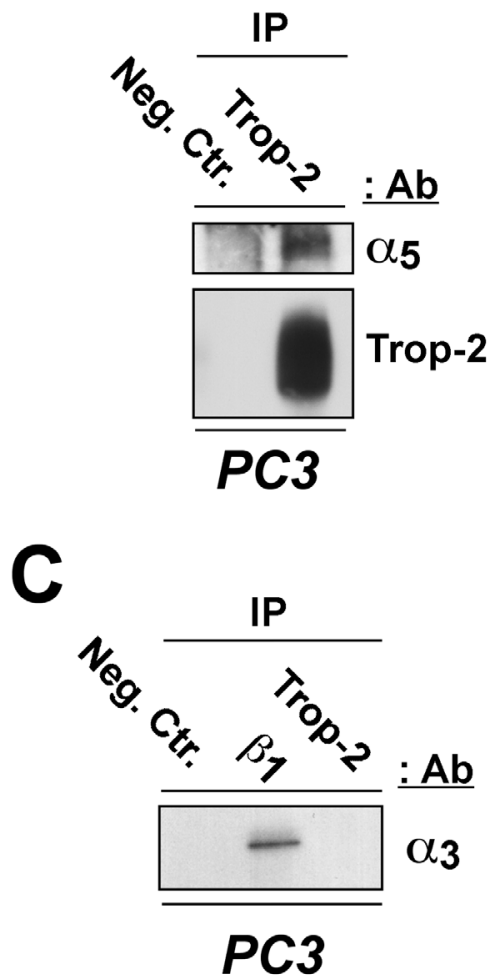

Figure 3: Correlation of Trop-2 and $\boldsymbol{\alpha 5} \boldsymbol{\beta} 1$ integrin expression in murine PCa. A. Analysis of $\alpha 5, \beta 1$ (top), $\alpha \mathrm{v}$ and $\beta 5$ (bottom) integrin subunits, as well as of Trop-2 (bottom) expression by IB using protein lysates from non-metastatic (left) and metastatic (right) prostate tumors collected from TRAMP mice. ERK1, control of protein loading. B. Protein lysates of PC3 cells endogenously expressing Trop-2 were immunoprecipitated using an Ab targeting Trop-2; a non-immune mouse IgG was used as a negative control Ab (Neg. Ctr.). The immunoprecipitates were then separated by SDS-PAGE and analyzed by IB for detection of the $\alpha 5$ integrin subunit and Trop-2. C. Protein lysates of PC 3 cells were immunoprecipitated using Abs targeting $\beta 1$ integrins or Trop-2; a non-immune mouse IgG was used as a negative control Ab (Neg. Ctr.). The immunoprecipitates were then analyzed by IB for detection of the $\alpha 3$ integrin subunit.

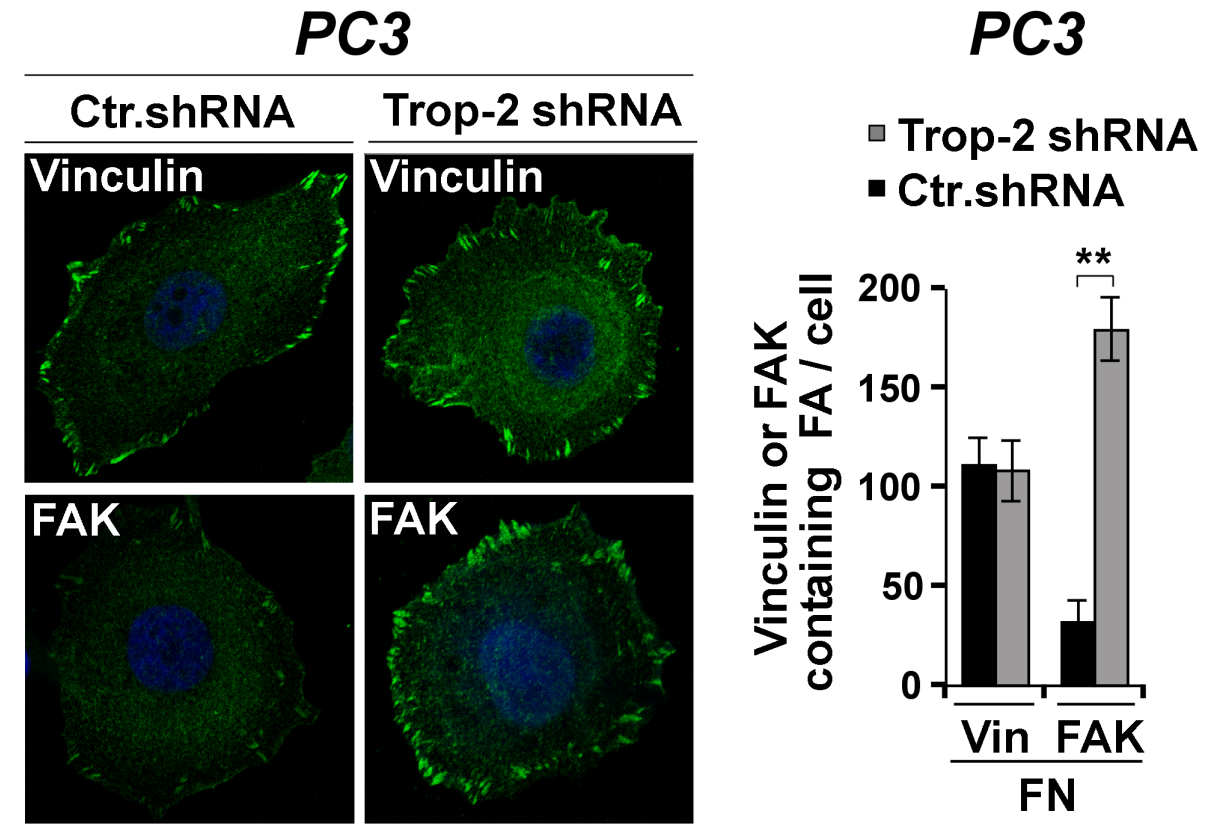

Figure 4: Trop-2-dependent modulation of FAK localization. Localization of vinculin and FAK in PC3/Ctr.shRNA and PC3/ Trop-2 shRNA cells seeded on FN was analyzed by IF (left). Vinculin (Vin)- and FAK-containing FAs were counted, and the average numbers per cell are shown in the bar graph (right). Error bars, SEM. ${ }^{* *}$, Student's $t$-test $P<0.001$. 


\section{Trop-2 is recruited in PCa exosomes that stimulate cell migration on $\mathrm{FN}$}

Release of exosomes from cancer cells has been shown to efficiently contribute to induction of metastatic dissemination by favoring intercellular communication $[14,24]$. We hypothesized that Trop-2 may be recruited to these cellular compartments, where $\beta 1$ integrins are also found [24-26]. Therefore, we isolated exosomes from PC3 culture supernatants and investigated by immunoblotting (IB) whether Trop-2 is recruited in these organelles as described in previous proteomic studies $[27,28]$. Exosome preparations were characterized by IB analysis of exosomal markers: CD63, CD81 (Figure 5B); as control, IB analysis of Calnexin was performed to
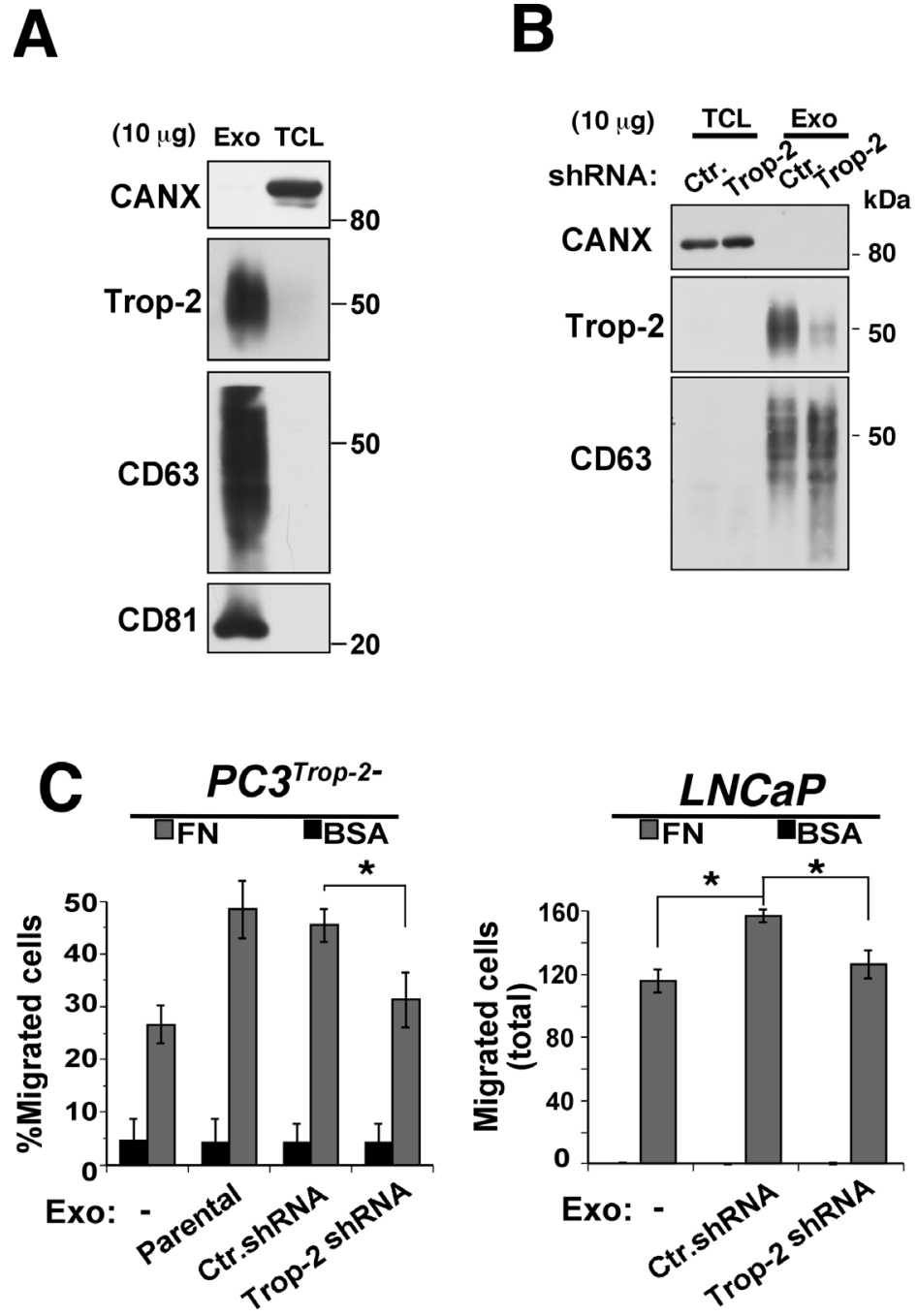

Figure 5: PC3 exosome uptake by PCa cells enhances cell migration on FN in a Trop-2-dependent manner. A. Analysis of Trop-2 levels in purified PC3 exosomal lysates separated by SDS-PAGE in non-reducing conditions and immunoblotted using an Ab to Trop-2; CD63 and CD81 were used as positive exosomal markers while calnexin (CANX) was used as a negative marker for exosomes. Exo, exosomes; TCL, total cell lysates. B. IB analysis of Trop-2 expression in exosomes secreted by PC3 cells (Ctr.shRNA and Trop-2 shRNA) using an Ab to Trop-2; CD63 was used as positive exosomal markers while calnexin (CANX) was used as a negative marker for

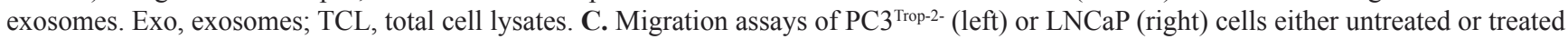
with $10 \mu \mathrm{g} / \mathrm{ml}$ of PC3 exosomes (Exo) in which Trop-2 is expressed (Parental and Ctr.shRNA) or down-regulated (Trop-2 shRNA). Left, $\chi^{2}$ test ; right, Student's $t$-test. *, $P \leq 0.05$. 


\section{DISCUSSION}

In this study, we demonstrate for the first time that Trop-2, an anti-adhesive and pro-migratory transmembrane protein, is up-regulated in human $\mathrm{PCa}$ with extracapsular extension (stages pT3/pT4) as compared to organconfined (stage pT2) PCa, suggesting that this molecule plays a crucial role during cancer progression toward a metastatic phenotype. Mechanistically, we show that Trop2 specifically binds the $\alpha 5 \beta 1$ integrin heterodimer and induces rearrangement of FA sites through displacement of FAK, thus perturbing the integrin signaling axis which is a major regulator of FA [29]. We finally find Trop-2 expression in exosomes secreted from $\mathrm{PCa}$ cells and demonstrate that Trop-2-containing exosomes stimulate migration of recipient Trop-2-negative cells on the $\alpha 5 \beta 1$ integrin substrate, FN.

The correlation between Trop-2 and disease progression suggests that this molecule is a novel biomarker of aggressive disease. During development, Trop-2 is expressed in the trophoblast, an actively invasive tissue at the interface between fetal and maternal circulation [30], whereas expression of this molecule in the adult is confined to a restricted number of tissues [21]. This pathway becomes exploited in malignancy, where Trop-2 is overexpressed in several human carcinomas, promotes accelerated tumor growth $[21,31]$, and correlates with unfavorable prognosis [1820]. Our findings reinforce a role of this pathway in the progression from organ-confined to disseminated cancer and this phenotype appears to be more insightful than Gleason scores in the histopathological analysis of stages pT2 and pT3/pT4. It remains to be investigated whether Trop-2 overexpression may be predictive of biochemical recurrence after prostatectomy as Gleason scores are poor predictors of recurrence [32].

We find that the up-regulation of Trop-2 in metastatic tumors from TRAMP mice correlates with expression of $\beta 1$ integrins (Figure $3 \mathrm{~A}$ ). While the mechanisms of Trop-2 up-regulation during disease progression are not known, we hypothesize that $\beta 1$ integrins and Trop2 expression may be co-regulated. Specifically, since previous studies from our group have shown that Insulinlike Growth Factor-1 Receptor (IGF-IR) plays a critical role in regulating $\beta 1$ integrin expression and since the absolute levels of IGF-IR increase during PCa progression in TRAMP tumors [33-35], we propose that IGF-IR may be an upstream regulator of Trop-2/ß1 integrin levels in metastasis-prone tumor types.

The proposed mechanism of metastatic dissemination, as suggested here, requires also upregulation of the $\alpha 5$ integrin subunit expression and appears specific since it does not affect the $\alpha 3$ integrin subunit. The acquisition of invasive potential by transformed cells has been linked to loss of E-cadherindependent cell-cell contacts, epithelial-to-mesenchymal transition [36] and consequent up-regulation of $\alpha 5$ [7]. Consistently, we observe that $\alpha 5$ is overexpressed in metastatic versus non-metastatic PCa using TRAMP mice as a model of disease progression. This may involve, in addition to the previously reported ability of $\alpha 5 \beta 1$ to promote epithelial-to-mesenchymal transition during cancer progression [7], a direct role in leading PCa invasion through recognition sequences in $\mathrm{FN}$ for the $\alpha 5$ subunit [37]. Our previous findings that the reduced adhesive phenotype induced by Trop-2 results in a higher rate of cell migration on FN [16] are consistent with a model of Trop- 2 modulation of $\alpha 5 \beta 1$ integrin signaling and selective displacement of FAK from FAs, that does not require changes in FAK expression as previously reported by our group [17].

We finally describe a novel mechanism involving accumulation of the Trop-2/ $\beta 1$ complex in secreted exosomes and demonstrate that exosomes containing Trop-2 impact neighboring tumor cells by enhancing their ability to migrate. These data suggest that therapeutics which interfere with the production, transfer or uptake of Trop-2-containing exosomes may attenuate tumor progression and metastasis.

Although more work is needed to fully elucidate the pro-invasive signaling pathways mediated by Trop-2 in $\mathrm{PCa}$ cells, the data presented here suggest that modulation of the Trop- $2 / \alpha 5 \beta 1$ complex may provide new insights in the functional stratification of PCa patients with higher metastatic propensity and, therefore, in need of more aggressive treatments.

\section{MATERIALS AND METHODS}

\section{Cells and culture conditions}

Cell lines and transfectants, as well as culture conditions have been described previously $[16,17]$. Authentication of the cell lines was provided with their purchase from American Type Culture Collection. PC3/ Ctr.shRNA and PC3/Trop-2 shRNA cells were generated as described previously $[16,21]$.

\section{Mice}

TRAMP mice, expressing SV40 large T antigen into the prostatic epithelium, were generated and characterized as described [23]. 23-54 week-old metastatic and nonmetastatic TRAMP mice were used to isolate tumor samples and perform IB analysis. Distant sites were: liver and lungs. All mice were maintained under specific pathogen-free conditions. Care and handling of animals was in compliance with IACUC experimental protocols. 


\section{Reagents and antibodies}

The T16 mouse monoclonal antibody (mAb) against Trop-2 (gift of Dr. S. Alberti) and the TS2/16 $\mathrm{mAb}$ against $\beta 1$ integrin (HB-243, ATCC) were used for IP. The following Abs were used for IB: mAbs against $\beta 1$ integrin subunit (610468, BD Transduction Laboratories), CD63 (Ab8219, Abcam), CD81 (Ab23505, Abcam); goat polyclonal Abs (pAbs) against human and murine Trop-2 (AF650 and AF1122, R\&D Biosystems); rabbit antisera against $\alpha 3$ and $\alpha \mathrm{v}$ integrin subunits; rabbit pAbs against $\alpha 5$ integrin subunit (sc-10729, Santa Cruz Biotechnology), $\beta 5$ integrin subunit (AB1926, Millipore), Calnexin (sc11397, Santa Cruz Biotechnology) and ERK1/2 (sc-93, Santa Cruz Biotechnology). The goat pAb against human Trop-2 (AF650, R\&D Biosystems), a mAb to Vinculin (MAB1624, Millipore) and a mAb to FAK (05-537, Millipore) were used for IF. A goat pAb against human Trop-2 (R\&D Biosystems) was used for IHC. Nonimmune goat $\operatorname{IgG}$ (SantaCruz), non-immune rabbit IgG (Sigma) and non-immune mouse IgG (Pierce) were used as negative control Abs. FN purification from human plasma has been previously described [17].

\section{Immunohistochemical analysis}

TMAs were constructed at the Vancouver Prostate Cancer Centre (Vancouver, Canada) from 74 men with newly diagnosed, previously untreated, clinically localized high-risk PCa, who underwent radical prostatectomy at the same Center. Ethical approval was obtained from the Institutional Ethical Review Board.

Specimens were identified for benign and cancerous sites and marked in donor paraffin blocks using matching H\&E-stained reference slides. The TMAs were constructed using a manual tissue microarrayer (Beecher Instruments, Silver Spring, MD, USA). Each marked block for benign and cancerous sites was sampled two times with a core diameter of $0.6 \mathrm{~mm}$ arrayed in a rectangular pattern with 1 $\mathrm{mm}$ between the center of each core, creating a duplicate TMA layout with a total of 148 cores. The TMA paraffin block was sectioned into 5- $\mu \mathrm{m}$ sections and mounted on positively charged slides.

IHC staining was conducted using a goat $\mathrm{pAb}$ to Trop-2 (AF650, R\&D Biosystems, dilution 1:25) by a Ventana autostainer model Discover XTTM (Ventana Medical System, Tuscan, AZ, USA) with an enzymelabelled biotin-streptavidin system and solvent-resistant 3,3'-diaminobenzidine Map kit. The slides were scanned on a BLISS system (Bacus Laboratory, North Lombard, IL, USA) and scored from 0 to +3 by a pathologist (L.F.) based on the staining intensity and the proportion of cells stained. Normal goat $\operatorname{IgG}$ was used as negative control Ab. All comparisons of staining intensities were made at 200X magnifications.

\section{Immunofluorescence and confocal microscopy}

Antigen retrieval was performed on rehydrated formalin-fixed paraffin-embedded sections from human or TRAMP mice PCa samples by incubation in $10 \mathrm{mM}$ Sodium Citrate Buffer (pH 6.0) at $95^{\circ} \mathrm{C}$ for $23 \mathrm{~min}$. The sections were blocked for $1 \mathrm{~h}$ at room temperature with PBS / 5\% BSA. Staining was performed by incubation of tissue samples with primary Abs (1:100) for $1 \mathrm{~h}$ at room temperature, followed by incubation with Alexa Fluor 633-Donkey anti goat (1:250) for $20 \mathrm{~min}$ at room temperature. Nuclei were counterstained using DAPI. After three washes, coverslips were mounted on the sections using Pro-Long anti-fade reagent (Invitrogen), and slides were analyzed on an inverted confocal microscope (LSM510, Carl Zeiss). Immunofluorescence analysis of PC3 cells using Alexa Fluor 488 goat antimouse (1:250) for $60 \mathrm{~min}$ at room temperature was performed as described [16].

\section{Generation of tumor lysates}

Tumor lysates were prepared by homogenizing the tissues on ice using the following lysis buffer: $100 \mathrm{mM}$ Tris- $\mathrm{HCl}$ (pH 7.4), $150 \mathrm{mM} \mathrm{NaCl}, 5 \%$ SDS, $0.1 \%$ Triton $\mathrm{X}-100,1 \mathrm{mM}$ benzamidine, $10 \mu \mathrm{g} / \mathrm{mL}$ Soybean Trypsin Inhibitor, $10 \mu \mathrm{g} / \mathrm{mL}$ leupeptin, $1 \mathrm{mM}$ PMSF, $1 \mu \mathrm{g} / \mathrm{mL}$ pepstatin $\mathrm{A}$, and $1 \mu \mathrm{M}$ calpain inhibitor. The lysates were boiled for 5 min and centrifuged at 13,000 rpm for 20 $\mathrm{min}$. Supernatants were collected and protein content was determined using the DC Protein Assay Kit (Bio-Rad). The protein samples (50 $\mu \mathrm{g}$ per lane) were separated by SDS-PAGE and transferred onto PVDF membranes for IB.

\section{Isolation and immunoblotting analysis of PCa exosomes}

Exosomes were isolated from $\mathrm{PCa}$ cells as described $[24,38]$. Purified exosomes were lysed with radioimmunoprecipitation assay buffer $(10 \mathrm{mM}$ Tris- $\mathrm{HCl}$ at $\mathrm{pH} 7.4,150 \mathrm{mM} \mathrm{NaCl}, 1 \mathrm{mM}$ EDTA, $0.1 \%$ SDS, $1 \%$ Triton X-100, and $1 \%$ sodium deoxycholate) supplemented with protease inhibitors. The protein samples $(10 \mu \mathrm{g}$ per lane) were separated by SDS-PAGE under non-reducing conditions and transferred onto PVDF membranes for IB.

\section{Immunoprecipitation}

To collect nuclear and cytoplasmic fractions, cells were washed with cold PBS and lysed by scraping in $20 \mathrm{mM}$ Tris- $\mathrm{HCl}$ (pH 7.4), $150 \mathrm{mM} \mathrm{NaCl}, 1 \mathrm{mM} \mathrm{CaCl}_{2}$, $1 \mathrm{mM} \mathrm{MgCl}, 1 \% \mathrm{NP}-40,1 \mathrm{mM}$ benzamidine, $10 \mu \mathrm{g} /$ $\mathrm{ml}$ leupeptin, $1 \mathrm{mM}$ PMSF, $1 \mu \mathrm{g} / \mathrm{ml}$ pepstatin $\mathrm{A}, 1 \mu \mathrm{M}$ calpain inhibitor, $1 \mathrm{mM} \mathrm{Na} \mathrm{VO}_{4}, 1 \mathrm{mM} \mathrm{Na} \mathrm{O}_{7} \mathrm{P}_{2}$. The 
cells were subjected to 3 cycles of sonication (15 sec each) on ice. After $15 \mathrm{~min}$ incubation on ice, lysates were centrifuged at $12,000 \mathrm{~g}$ for $10 \mathrm{~min}$, and supernatants were collected and pre-cleared by two consecutive incubations with protein $\mathrm{G}$-Sepharose at $4^{\circ} \mathrm{C}$ for $45 \mathrm{~min}$. Binding to specific Abs was performed by incubation at $4^{\circ} \mathrm{C}$ for $3 \mathrm{~h}$, followed by incubation with protein G-Sepharose at $4^{\circ} \mathrm{C}$ for $1 \mathrm{~h}$. After six washes with lysis buffer, immunocomplexes were eluted with $100 \mathrm{mM}$ glycine $\mathrm{pH}$ 2.5 , followed by $\mathrm{pH}$ neutralization using Tris to a final concentration of $50 \mathrm{mM}$. The immunocomplexes were then separated by SDS-PAGE, transferred onto PVDF membrane, and subjected to analysis by IB.

\section{Exosome treatment and migration assay}

Cell treatment with exosomes was performed as previously described [24]. Briefly, LNCaP and PC3 $3^{\text {Trop-2- }}$ cells were serum starved for $18 \mathrm{~h}$, then treated for $24 \mathrm{~h}$ with $20 \mu \mathrm{g} / \mathrm{ml}$ of exosomes obtained from PC3 cells. The cells treated with PC3-derived exosomes were trypsinized, extensively washed with PBS and subsequently plated to perform the migration assay. Cell migration assays on FN has been performed as previously described using Millicell inserts (Millipore) with 8 (for LNCaP) or 12 $\mu \mathrm{m}$ (for PC3 ${ }^{\text {Trop-2-) }}$ pores [16]. Briefly, chambers were coated on both top and bottom layers with FN (10 $\mu \mathrm{g} /$ $\mathrm{mL}$ ) or $1 \% \mathrm{BSA}$ overnight at $4^{\circ} \mathrm{C}$. After cell detachment and trypsin inactivation, cells were seeded on coated transwell chambers at $37^{\circ} \mathrm{C}$ for $6 \mathrm{~h}$. After fixation with $3.7 \%$ paraformaldehyde (PFA), cells attached on both layers of the porous filter were stained with $1 \mu \mathrm{g} / \mathrm{mL}$ 4',6-diamidino-2-phenylindole (DAPI) and pictures of nuclei were acquired by fluorescence microscopy. Then, cells on the top layer were removed using a cotton swab, and pictures of nuclei from cells migrated to the bottom layer were acquired. For each group of PC $3^{\text {Trop-2- cells }}$ treated with or without exosomes, the ratio between number of cells migrated onto the bottom layer and total (top + bottom) number of cells attached on the filter was calculated. For LNCaP cells, only the total number of cells attached on the bottom layer was calculated.

\section{Statistical analysis}

For patients' samples, Fisher's exact test was used to examine the association between dichotomized biomarkers (Trop-2) and PCa stage (Gleason score). For FA analysis, $t$-test was used to evaluate the average numbers of FA per cell between PC3/Ctr.shRNA and PC3/Trop-2 shRNA cells. For migration assays $\chi^{2}$ tests and $t$-test were used to compare the migration between treatments. Stata 12.0 (StatCORP LP, College Station, TX, USA was used for data analysis $P \leq 0.05$ was considered as statistical significance.

\section{ACKNOWLEDGMENTS}

We thank Dr. S. Alberti for providing the $\mathrm{mAb}$ (clone T16) against Trop-2 and Dr. L. Borgia for helpful discussion. The authors would like to thank Dr. A. Sayeed, as well as R. M. De Rita, A. N. Duffy and A. Singh in Languino's lab for constructive suggestions. We are also grateful to Mrs. M. Harden and Mrs. C. Deemer for their help with the preparation of the manuscript.

\section{GRANT SUPPORT}

This work was supported by NIH (LRL, DCA), NIH-R01CA109874 (LRL), NIH-R01CA089720 (LRL), NIH-R01CA086072 (RGP); the Office of the Assistant Secretary of Defense for Health Affairs through the Prostate Cancer Research Program under Award No. W81XWH-13-1-0193 (DCA), and by a Post-Doctoral Research Fellowship from the Italian Association for Cancer Research to M.T. and by a Post-Doctoral Research Fellowship from the American Italian Cancer Foundation to C.F. Research in this publication includes work carried out using the Sidney Kimmel Cancer Center Bioimaging Facility and the Translational Research \& Pathology Shared Resource, which are supported in part by NCI Cancer Center Support Grant P30 CA56036 (RGP). This project is also funded, in part, under a Commonwealth University Research Enhancement Program grant with the Pennsylvania Department of Health (H.R.); the Department specifically disclaims responsibility for any analyses, interpretations or conclusions.

\section{CONFLICTS OF INTEREST}

The Authors do not have any conflicts of interest.

\section{Abbreviations}

PCa, prostate cancer; ECM, extracellular matrix; TNM, tumor, node and metastasis; PSA, prostate specific antigen; TRAMP, TRansgenic Adenocarcinoma of Mouse Prostate; IB, immunoblotting; mAb, monoclonal antibody; pAb, polyclonal antibody; IF, immunofluorescence; IHC, immunohistochemistry; TMA, Tissue Microarray; FAK, focal adhesion kinase; FN, fibronectin.

\section{REFERENCES}

1. Edge SB and American Joint Committee on Cancer. (2010). AJCC cancer staging manual. (New York: Springer).

2. Sobin LH, Gospodarowicz MK, Wittekind $\mathrm{C}$ and Cancer IUa. (2010). TNM classification of malignant tumours. (Chichester, West Sussex, UK ; Hoboken, NJ: WileyBlackwell).

3. Logothetis CJ, Gallick GE, Maity SN, Kim J, Aparicio 
A, Efstathiou E and Lin SH. Molecular classification of prostate cancer progression: foundation for marker-driven treatment of prostate cancer. Cancer Discov. 2013; 3:849861.

4. Knox JD, Cress AE, Clark V, Manriquez L, Affinito KS, Dalkin BL and Nagle RB. Differential expression of extracellular matrix molecules and the alpha 6-integrins in the normal and neoplastic prostate. Am J Pathol. 1994; 145:167-174.

5. Murant SJ, Handley J, Stower M, Reid N, Cussenot O and Maitland NJ. Co-ordinated changes in expression of cell adhesion molecules in prostate cancer. Eur J Cancer. 1997; $33: 263-271$.

6. Goel HL, Underwood JM, Nickerson JA, Hsieh CC and Languino LR. $\beta 1$ integrins mediate cell proliferation in three-dimensional cultures by regulating expression of the sonic hedgehog effector protein, GLI1. J Cell Physiol. 2010; 224:210-217.

7. Sawada K, Mitra AK, Radjabi AR, Bhaskar V, Kistner EO, Tretiakova M, Jagadeeswaran S, Montag A, Becker A, Kenny HA, Peter ME, Ramakrishnan V, Yamada SD and Lengyel E. Loss of E-cadherin promotes ovarian cancer metastasis via $\alpha 5$-integrin, which is a therapeutic target. Cancer Res. 2008; 68:2329-2339.

8. Adachi M, Taki T, Higashiyama M, Kohno N, Inufusa H and Miyake M. Significance of integrin $\alpha 5$ gene expression as a prognostic factor in node-negative non-small cell lung cancer. Clin Cancer Res. 2000; 6:96-101.

9. Dingemans AM, van den Boogaart V, Vosse BA, van Suylen RJ, Griffioen AW and Thijssen VL. Integrin expression profiling identifies integrin $\alpha 5$ and $\beta 1$ as prognostic factors in early stage non-small cell lung cancer. Mol Cancer. 2010; 9:152.

10. Kuwada SK and Li X. Integrin $\alpha 5 / \beta 1$ mediates fibronectindependent epithelial cell proliferation through epidermal growth factor receptor activation. Mol Biol Cell. 2000; 11:2485-2496.

11. Morozevich G, Kozlova N, Cheglakov I, Ushakova N and Berman A. Integrin $\alpha 5 \beta 1$ controls invasion of human breast carcinoma cells by direct and indirect modulation of MMP2 collagenase activity. Cell Cycle. 2009; 8:2219-2225.

12. Akiyama SK. Integrins in cell adhesion and signaling. Hum Cell. 1996; 9:181-186.

13. Luga V, Zhang L, Viloria-Petit AM, Ogunjimi AA, Inanlou MR, Chiu E, Buchanan M, Hosein AN, Basik M and Wrana JL. Exosomes mediate stromal mobilization of autocrine Wnt-PCP signaling in breast cancer cell migration. Cell. 2012; 151:1542-1556.

14. Peinado H, Aleckovic M, Lavotshkin S, Matei I, CostaSilva B, Moreno-Bueno G, Hergueta-Redondo M, Williams C, Garcia-Santos G, Ghajar C, Nitadori-Hoshino A, Hoffman C, Badal K, Garcia BA, Callahan MK, Yuan J, et al. Melanoma exosomes educate bone marrow progenitor cells toward a pro-metastatic phenotype through MET. Nat
Med. 2012; 18:883-891.

15. Valadi H, Ekstrom K, Bossios A, Sjostrand M, Lee JJ and Lotvall JO. Exosome-mediated transfer of mRNAs and microRNAs is a novel mechanism of genetic exchange between cells. Nat Cell Biol. 2007; 9:654-659.

16. Trerotola M, Jernigan DL, Liu Q, Siddiqui J, Fatatis A and Languino LR. Trop-2 promotes cancer metastasis by modulating $\beta 1$ integrin functions. Cancer Res. 2013; 73:3155-3167.

17. Trerotola M, Li J, Alberti S and Languino LR. Trop2 inhibits prostate cancer cell adhesion to fibronectin through the $\beta 1$ integrin-RACK1 axis. J Cell Physiol. 2012; 227:3670-3677.

18. Fong D, Moser P, Krammel C, Gostner JM, Margreiter R, Mitterer M, Gastl G and Spizzo G. High expression of TROP2 correlates with poor prognosis in pancreatic cancer. Br J Cancer. 2008; 99:1290-1295.

19. Kluger HM, Kluger Y, Gilmore-Hebert M, DiVito K, Chang JT, Rodov S, Mironenko O, Kacinski BM, Perkins AS and Sapi E. cDNA microarray analysis of invasive and tumorigenic phenotypes in a breast cancer model. Lab Invest. 2004; 84:320-331.

20. Ohmachi T, Tanaka F, Mimori K, Inoue H, Yanaga K and Mori M. Clinical significance of TROP2 expression in colorectal cancer. Clin Cancer Res. 2006; 12:3057-3063.

21. Trerotola M, Cantanelli P, Guerra E, Tripaldi R, Aloisi AL, Bonasera V, La Sorda R, Lattanzio R, de Lange R, Weidle U, Piantelli $\mathrm{M}$ and Alberti S. Upregulation of Trop-2 quantitatively stimulates human cancer growth. Oncogene. 2012; 32:222-233.

22. Ellis L, Lehet K, Ku S, Azabdaftari G and Pili R. Generation of a syngeneic orthotopic transplant model of prostate cancer metastasis. Oncoscience. 2014; 1:609-613.

23. Greenberg NM, DeMayo F, Finegold MJ, Medina D, Tilley WD, Aspinall JO, Cunha GR, Donjacour AA, Matusik RJ and Rosen JM. Prostate cancer in a transgenic mouse. Proc Natl Acad Sci USA. 1995; 92:3439-3443.

24. Fedele C, Singh A, Zerlanko BJ, Iozzo RV and Languino LR. The $\alpha v \beta 6$ Integrin Is Transferred Intercellularly via Exosomes. J Biol Chem. 2015; 290:4545-4551.

25. Bijnsdorp IV, Geldof AA, Lavaei M, Piersma SR, van Moorselaar RJ and Jimenez CR. Exosomal ITGA3 interferes with non-cancerous prostate cell functions and is increased in urine exosomes of metastatic prostate cancer patients. J Extracell Vesicles. 2013; 2.

26. Clayton A, Turkes A, Dewitt S, Steadman R, Mason MD and Hallett MB. Adhesion and signaling by B cell-derived exosomes: the role of integrins. FASEB J. 2004; 18:977979.

27. Chen CL, Lai YF, Tang P, Chien KY, Yu JS, Tsai CH, Chen HW, Wu CC, Chung T, Hsu CW, Chen CD, Chang YS, Chang PL and Chen YT. Comparative and targeted proteomic analyses of urinary microparticles from bladder cancer and hernia patients. J Proteome Res. 2012; 11:5611- 
5629.

28. Tauro BJ, Greening DW, Mathias RA, Mathivanan S, Ji $\mathrm{H}$ and Simpson RJ. Two distinct populations of exosomes are released from LIM1863 colon carcinoma cell-derived organoids. Mol Cell Proteomics. 2013; 12:587-598.

29. Cox BD, Natarajan M, Stettner MR and Gladson CL. New concepts regarding focal adhesion kinase promotion of cell migration and proliferation. J Cell Biochem. 2006; 99:3552.

30. Lipinski M, Parks DR, Rouse RV and Herzenberg LA. Human trophoblast cell-surface antigens defined by monoclonal antibodies. Proc Natl Acad Sci USA. 1981; 78:5147-5150.

31. Guerra E, Trerotola M, Dell' Arciprete R, Bonasera V, Palombo B, El-Sewedy T, Ciccimarra T, Crescenzi C, Lorenzini F, Rossi C, Vacca G, Lattanzio R, Piantelli M and Alberti S. A bicistronic CYCLIN D1-TROP2 mRNA chimera demonstrates a novel oncogenic mechanism in human cancer. Cancer Res. 2008; 68:8113-8121.

32. Bedolla R, Prihoda TJ, Kreisberg JI, Malik SN, Krishnegowda NK, Troyer DA and Ghosh PM. Determining risk of biochemical recurrence in prostate cancer by immunohistochemical detection of PTEN expression and Akt activation. Clin Cancer Res. 2007; 13:3860-3867.

33. Goel H, Sayeed A, Breen M, Zarif MJ, Garlick DS, Leav I, Davis RJ, Fitzgerald TJ, Morrione A, Liu Q, Dicker A, Altieri DC and Languino LR. $\beta 1$ integrins mediate resistance to ionizing radiation by inhibiting JNK. J Cell Physiol. 2013; 228:1601-1609.

34. Sutherland BW, Knoblaugh SE, Kaplan-Lefko PJ, Wang F, Holzenberger $\mathrm{M}$ and Greenberg NM. Conditional deletion of insulin-like growth factor-I receptor in prostate epithelium. Cancer Res. 2008; 68:3495-3504.

35. Goel HL, Breen M, Zhang J, Das I, Aznavoorian-Cheshire S, Greenberg NM, Elgavish A and Languino LR. $\beta 1 \mathrm{~A}$ integrin expression is required for type 1 insulin-like growth factor receptor mitogenic and transforming activities and localization to focal contacts. Cancer Res. 2005; 65:66926700 .

36. Hanahan D and Weinberg RA. Hallmarks of cancer: the next generation. Cell. 2011; 144:646-674.

37. Aota S, Nomizu M and Yamada KM. The short amino acid sequence Pro-His-Ser-Arg-Asn in human fibronectin enhances cell-adhesive function. J Biol Chem. 1994; 269:24756-24761.

38. Thery C, Amigorena S, Raposo G and Clayton A. (2006). Isolation and characterization of exosomes from cell culture supernatants and biological fluids (John Wiley \& Sons, Inc). 